\title{
Quasars near the line of sight towards Q 0302-003 and the transverse proximity effect ${ }^{\star}$
}

\author{
G. Worseck and L. Wisotzki
}

Astrophysikalisches Institut Potsdam, An der Sternwarte 16, 14482 Potsdam, Germany

e-mail: gworseck@aip.de

Received 18 November 2005 / Accepted 16 January 2006

\section{ABSTRACT}

We report the discovery of the faint $(V \simeq 21.7)$ quasar QSO $03027-0010$ at $z=2.808$ in the vicinity of Q 0302-003, one of the few quasars observed with STIS to study intergalactic He II absorption. Together with another newly discovered QSO at $z=2.29$, there are now 6 QSOs known near the line of sight towards Q 0302-003, of which 4 are located within the redshift region $2.76 \lesssim z \lessgtr 3.28$ covered by the STIS spectrum. We correlated the opacity variations in the H I and He II Lyman forest spectra with the locations of known quasars. There is no significant proximity effect in the H I Ly $\alpha$ forest for any of the QSOs, except for the well-known line of sight effect for Q 0302-003 itself. By comparing the absorption properties in $\mathrm{HI}$ and He II, we estimated the fluctuating hardness of the extragalactic UV radiation field along this line of sight. We find that close to each foreground quasar, the ionizing background is considerably harder than on average. In particular, our newly discovered QSO 03027-0010 shows such a hardness increase despite being associated with an overdensity in the H I Lyman forest. We argue that the spectral hardness is a sensitive physical measure to reveal the influence of QSOs onto the UV background even over scales of several $\mathrm{Mpc}$, and that it breaks the density degeneracy hampering the traditional transverse proximity effect analysis. We infer from our sample that there is no need for significantly anisotropic UV radiation from the QSOs. From the transverse proximity effect detected in the sample we obtain minimum quasar lifetimes in the range $\sim 10-30$ Myr.

Key words. quasars: general - quasars: absorption lines - intergalactic medium - diffuse radiation

\section{Introduction}

Observations of high-redshift quasars enable us to study the intergalactic medium (IGM) along their lines of sight via the absorption of quasar radiation by various chemical elements in different ionization stages. Hydrogen and helium are by far the most abundant elements in the universe and the Ly $\alpha$ transitions of $\mathrm{H} \mathrm{I}$ and $\mathrm{He}$ II in an incompletely ionized medium cause a Gunn-Peterson trough at redshifts smaller than the emission redshift of the observed quasar (Gunn \& Peterson 1965). If the IGM is highly ionized, a plethora of discrete absorption lines stemming from the remaining neutral fraction is visible both in H I Ly $\alpha$ and He II Ly $\alpha$, giving rise to the name Ly $\alpha$ forest.

The intensity of the metagalactic UV radiation field at a characteristic frequency (typically the ionization energy of a given element) varies in time due to the temporal evolution

^ Based on observations collected at the European Southern Observatory, Chile (Proposals 070.A-0425 and 074.A-0273). Data collected under Proposal 068.A-0194 was obtained from the ESO Science Archive. Based on observations made with the NASA/ESA Hubble Space Telescope, obtained from the data archive at the Space Telescope Institute. STScI is operated by the association of Universities for Research in Astronomy, Inc. under the NASA contract NAS 5-26555. of the source population. Spatial fluctuations induced by the discreteness of the source population have been examined for randomly distributed sources and absorbers by Zuo (1992) and Fardal \& Shull (1993). They are also treated in recent numerical simulations trying to quantify their impact on the Ly $\alpha$ flux power spectrum (Croft et al. 1999; Meiksin \& White 2004; Croft 2004; McDonald et al. 2005). The spatial fluctuations of the UV radiation field computed from these simulations along random lines of sight are generally gentle (few per cent around the mean) and occur on large scales ( $\gtrsim 100$ Mpc comoving) at $z<4$. This simple picture with only mild large-scale fluctuations is expected to change considerably for lines of sight that pass close to sources of the UV background, such as luminous quasars (Fardal \& Shull 1993; Croft 2004; McDonald et al. 2005). The source flux acts as a local enhancement of the UV radiation field. As a consequence, the absorbers in the region affected by this excess flux will be statistically more ionized than the rest of the Ly $\alpha$ forest along the line of sight, resulting in a statistically increased transmission, a radiation-induced "void" in the H I Ly $\alpha$ forest in the vicinity of the UV source. This so-called proximity effect has been detected with high statistical significance in lines of sight towards luminous quasars (e.g. Bajtlik et al. 1988; Scott et al. 2000). However, a transverse proximity effect created by foreground ionizing sources 
nearby the line of sight has not been clearly detected in the H I Ly $\alpha$ forest. The full range of possible results extends from large voids claimed to be due to the transverse proximity effect by Dobrzycki \& Bechtold (1991a; however see Dobrzycki $\&$ Bechtold 1991b) and Srianand (1997), over marginal detections (Fernández-Soto et al. 1995; Liske \& Williger 2001) to non-detections (Crotts 1989; Møller \& Kjærgaard 1992; Crotts \& Fang 1998; Schirber et al. 2004). Croft (2004) measured the average transverse Ly $\alpha$ transmission from all projected quasar pairs in the SDSS DR1 and even found excess absorption near foreground quasars instead of the expected excess transmission caused by the transverse proximity effect.

The detectability of the transverse proximity effect is hampered by several systematic effects. Anisotropic radiation of quasars has been invoked to explain redshift offsets between the void and the foreground quasar (Dobrzycki \& Bechtold 1991a) or to explain the lack of the transverse proximity effect (Crotts 1989; Møller \& Kjærgaard 1992; Schirber et al. 2004). Also quasar variability affects the detection of the proximity effect (Schirber et al. 2004). Finally, the possible gravitational clustering around quasars that are assumed to reside in the densest environments may dilute the proximity effect (Loeb \& Eisenstein 1995; Schirber et al. 2004; Rollinde et al. 2005). According to Schirber et al. (2004) only a combination of these systematic effects may explain the apparent absence of the transverse proximity effect.

He II Ly $\alpha 303.78 \AA$ absorption can be studied only towards a few lines of sight to date because most of the quasars at $z>2$ have intervening optically thick Lyman limit systems that truncate the flux in the observable He II Ly $\alpha$ wavelength range in the far UV. The observations of the lines of sight towards Q 0302-003 at $z=3.285$ (Jakobsen et al. 1994; Hogan et al. 1997; Heap et al. 2000), PKS 1935-692 at $z=3.18$ (Anderson et al. 1999) and recently SDSS J2346-0016 at $z=3.50$ (Zheng et al. 2004a) show in most parts of their He II absorption spectra very strong absorption at $z>3$ that is consistent with a Gunn-Peterson trough $\left(\tau_{\mathrm{He} \text { II }}>3\right)$. In contrast, the three lines of sight at $z<3$ probed so far towards HS $1700+6416$ at $z=2.72$ (Davidsen et al. 1996; Reimers et al. 2004), HE 2347-4342 at $z=2.885$ (Reimers et al. 1997; Kriss et al. 2001; Smette et al. 2002; Shull et al. 2004; Zheng et al. 2004b) and recently QSO $1157+3143$ at $z \simeq 3$ (Reimers et al. 2005) display patchy intergalactic He II absorption with voids $\left(\tau_{\mathrm{He} \mathrm{II}} \lesssim 1\right)$ and troughs $\left(\tau_{\mathrm{He} \text { II }}>3\right)$ that evolves to a He II Ly $\alpha$ forest at $z<2.7$ resolved with FUSE (Kriss et al. 2001; Shull et al. 2004; Zheng et al. 2004b; Reimers et al. 2004). In combination with the observed evolution of H I line widths (Schaye et al. 2000; Ricotti et al. 2000; Theuns et al. 2002) these observations point to a late He II reionization between $z \sim 2.7$ and $z \sim 3$.

By comparing the $\mathrm{HI}$ absorption with the corresponding He II absorption one can estimate the hardness of the ionizing radiation field that penetrates the IGM, since $\mathrm{HI}$ is ionized at $h v>13.6 \mathrm{eV}$, whereas He II is ionized at $h v>54.4 \mathrm{eV}$. The amount of He II compared to H I gives a measure of the spectral hardness. Already low-resolution He II observations obtained with HST indicated a fluctuating radiation field in the voids (hard) and the troughs (soft) (Reimers et al. 1997; Heap et al. 2000; Smette et al. 2002). The recent high-resolution FUSE observations of the He II Ly $\alpha$ forest reveal large fluctuations on very small scales of $\Delta z \sim 10^{-3}$ (Kriss et al. 2001; Shull et al. 2004; Reimers et al. 2004). Due to the hard ionizing field required in the He II voids that is consistent with the integrated radiation of a surrounding quasar population, these He II voids have been interpreted as the onset of $\mathrm{He}$ II reionization in Strömgren spheres around hard He II photoionizing sources along or near the line of sight (Reimers et al. 1997; Heap et al. 2000; Smette et al. 2002). A subsequent survey for putative quasars that may cause the prominent He II void at $z=3.05$ towards Q 0302-003 yielded the quasar QSO 03020-0014 located 6.5 away on the sky that coincides with this He II void (Jakobsen et al. 2003). Thus, the He II void in Q 0302-003 is the first clear case of a transverse proximity effect due to a luminous quasar.

In this paper we report on results from a slitless spectroscopic quasar survey that resulted in the discovery of another foreground quasar in the vicinity of Q 0302-003. The structure of the paper is as follows. Section 2 describes the observation and the supplementary data employed for the paper. In Sects. 3 and 4 we examine the evidence for opacity variations in the $\mathrm{HI}$ and He II Ly $\alpha$ forest regions caused by intensity fluctuations in the UV radiation field towards Q 0302-003 at 1 ryd and 4 ryd, respectively. In Sect. 5 we consider the possibility to detect the transverse proximity effect via spectral hardness diagnostics of the ionizing radiation. We argue that this is actually the most sensitive method, and we demonstrate that essentially each quasar near the line of sight is associated with a local hardening of the radiation field. Finally, we use these observations to estimate a lower limit to the quasar lifetime (Sect. 7). We present our conclusions in Sect. 8. Throughout the paper we adopt a flat cosmological model with $\Omega_{\mathrm{m}}=0.3, \Omega_{\Lambda}=0.7$ and $H_{0}=70 \mathrm{~km} \mathrm{~s}^{-1} \mathrm{Mpc}^{-1}$.

\section{Observations and data reduction}

\subsection{Search for quasar candidates near Q 0302-003}

In October 2002 and February 2003 we conducted a quasar survey with the ESO Wide Field Imager (WFI) at the ESO/MPI $2.2 \mathrm{~m}$ Telescope at La Silla in its slitless spectroscopic mode (Wisotzki et al. 2001). The survey fields of $25^{\prime} \times 33^{\prime}$ were centered on bright high-redshift QSOs, and our aim was to find faint quasars in their vicinity. Details about this survey will be given in a separate paper. Here we report the results of the field centered on Q 0302-003 at $z=3.285$ observed on October 3 and 4, 2002, and we only briefly summarize the survey technique. We recorded our slitless spectra with the R50 grism $(\lambda / \Delta \lambda=30-50$ depending on the seeing) in the $B$ and the $V$ band by 3 dithered 600 s exposures in 2 instrument rotations each, resulting in a total exposure time of $1 \mathrm{~h}$ per band and yielding a limiting magnitude of $V_{\lim } \simeq 22$. The filters were employed to reduce the sky background and the degree of crowding by limiting the length of the recorded spectra. Crowding was further accounted for by taking the spectra in two instrument rotations $\left(0^{\circ}\right.$ and $\left.10^{\circ}\right)$. The combination of the R50 grism with the broadband $B$ and $V$ filters resulted in a spectral coverage from the blue grism sensitivity cutoff at $4200 \AA$ 

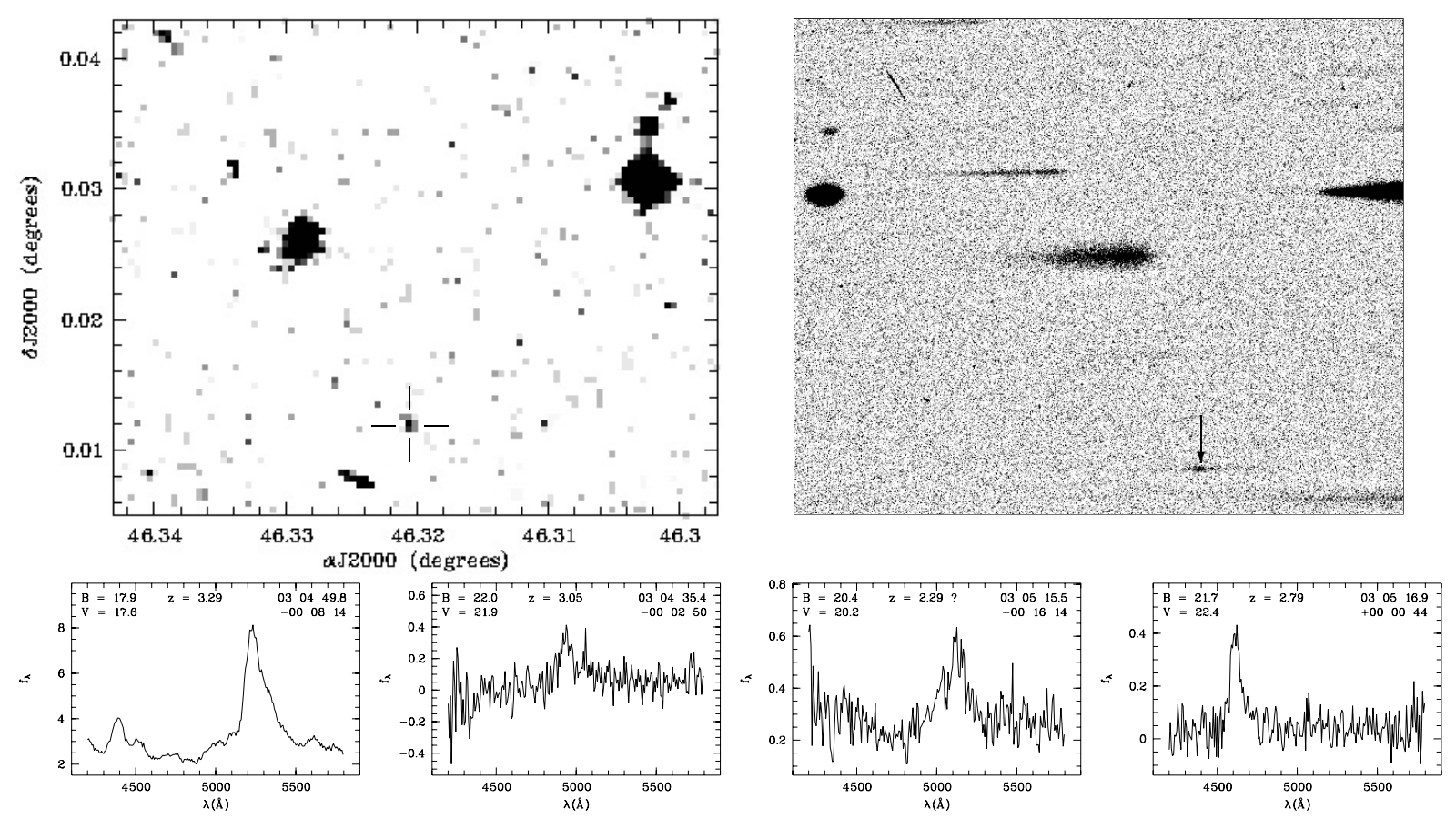

Fig. 1. Discovery of the two quasar candidates. The upper left panel shows a $2.8^{\prime} \times 2.3^{\prime}$ DSS1 image of the sky region around the faint QSO 03027-0010 marked with a cross. The upper right panel displays the corresponding region of the slitless WFI $B$ exposures $\left(0^{\circ}\right.$ rotation, $1800 \mathrm{~s}$ stacked observations). The emission line of QSO 03027-0010 is clearly visible (arrow). The lower panels show the combined and calibrated slitless spectra of $z>2$ quasars found on the total $25^{\prime} \times 33^{\prime}$ field with their DSS1 position, redshift and magnitude estimates (from left to right: Q 0302-003, QSO 03020-0014, QSO 03027-0027 and QSO 03027-0010).

to $5800 \AA$ A permitting us to search for $\operatorname{Ly} \alpha$ emission in the redshift range $2.46 \lesssim z \lesssim 3.77$ and for $\mathrm{C}$ IV emission in the range $1.71 \lesssim z \lesssim 2.74$.

All sources detected in the Digitized Sky Survey (which has approximately the same depth as our slitless spectroscopic data) were automatically extracted, yielding flux-calibrated low-resolution spectra of $\sim 800$ objects in the field. The spectra were searched automatically for emission-line objects. The lower panel of Fig. 1 shows the slitless spectra of four $z>2$ quasars that were found by our survey. One of them was (unsurprisingly) Q 0302-003 itself, and we also unambiguously rediscovered QSO 03020-0014 discovered recently by Jakobsen et al. (2003). Our search revealed two further quasar candidates in the vicinity of Q 0302-003 that fall into our redshift range of interest. The first showed clearly Ly $\alpha$ emission at $z \simeq 2.79$ whereas the second displayed an emission line which we tentatively identified as C IV at $z \simeq 2.29$.

\subsection{Spectroscopic follow-up}

Follow-up spectroscopy of these two quasar candidates was obtained with the Focal Reducer/Low Dispersion Spectrograph 2 (FORS2) on ESO VLT UT1/Antu in Visitor Mode on November 17, 2004. The sky was clear throughout the night. The spectra were taken with the $300 \mathrm{~V}$ grism and a $1^{\prime \prime}$ slit kept at the parallactic angle, resulting in a spectral resolution of $\sim 10 \AA F W H M$. In order to maximize throughput, the order separation filter was omitted, leading to possible order overlap at $\lambda>6600 \AA$. Exposure times were adjusted to yield a $S / N$ of $\sim 20$ in the quasar continuum.
We also recorded a FORS2 spectrum of Q 0302-003 with the 600B grism on November 19, 2004, at a resolution of $\sim 4.5 \AA F W H M$. Seeing conditions were poor and highly variable during the observations of $Q$ 0302-003, resulting in some slit losses but still a $S / N$ of $\sim 70$ in the Ly $\alpha$ forest could be achieved. The spectra were calibrated in wavelength against the FORS2 $\mathrm{He} / \mathrm{Ne} / \mathrm{Ar} / \mathrm{HgCd}$ arc lamps and spectrophotometrically calibrated against the HST standard stars Feige 110 and GD 108. Data reduction was performed with standard IRAF tasks. The spectra were optimally extracted using the algorithm introduced by Horne (1986). Table 1 summarizes our spectroscopic follow-up observations.

Figure 2 shows the calibrated spectra of the two candidates. Both are confirmed to be quasars at redshifts correctly estimated in the slitless spectra. Since the quality of our FORS2 aquisition images is superior to the DSS image, we refined the QSO positions (see Table 1) and denote these as QSO 03027-0010 and QSO 03027-0027 in the following, according to IAU conventions.

There is a strong associated absorption line system in the spectrum of QSO 03027-0010 at $z_{\mathrm{abs}}=2.804 \pm 0.001$ visible in Ly $\alpha, \mathrm{N} \mathrm{V}$ and C IV. A C IV system is present at $z_{\mathrm{abs}}=2.696$ that is also visible in Si IV and that corresponds to a strong Ly $\alpha$ absorption line in the Ly $\alpha$ forest. Moreover, a Mg II absorber may be present at $z_{\mathrm{abs}}=0.750$.

QSO 03027-0027 shows a strong metal line system at $z_{\text {abs }}=2.115$, easily detectable in Mg II, C IV, Si IV and C II. Another weaker C IV system may be present at $z_{\mathrm{abs}}=2.243$.

Table 2 shows our quasar redshift determination based on all detectable emission lines. The $S / N$ of both spectra generally 
Table 1. Observing log for the spectroscopic follow-up.

\begin{tabular}{lllllllll}
\hline \hline Object & $\alpha(\mathrm{J} 2000)$ & $\delta(\mathrm{J} 2000)$ & Night & Grism & Slit & Exposure & Airmass & Seeing \\
\hline QSO 03027-0010 & $03^{\mathrm{h}} 05^{\mathrm{m}} 16.80$ & $+00^{\circ} 00^{\prime} 45^{\prime \prime} .1$ & 17 Nov. 2004 & $300 \mathrm{~V}$ & $1^{\prime \prime} 0$ & $600 \mathrm{~s}$ & 1.10 & $0 !^{\prime} 9$ \\
QSO 03027-0027 & $03^{\mathrm{h}} 05^{\mathrm{m}} 15^{\mathrm{s}} .62$ & $-00^{\circ} 16^{\prime} 14^{\prime \prime} .4$ & 17 Nov. 2004 & $300 \mathrm{~V}$ & $1^{\prime \prime} 0$ & $300 \mathrm{~s}$ & 1.10 & 0.7 \\
Q 0302-003 & $03^{\mathrm{h}} 04^{\mathrm{m}} 49.71$ & $-00^{\circ} 08^{\prime} 13^{\prime \prime} 0$ & 19 Nov. 2004 & $600 \mathrm{~B}$ & $1^{\prime \prime} 0$ & $1800 \mathrm{~s}$ & 1.18 & $\$ 1^{\prime \prime} 9$ \\
\hline
\end{tabular}

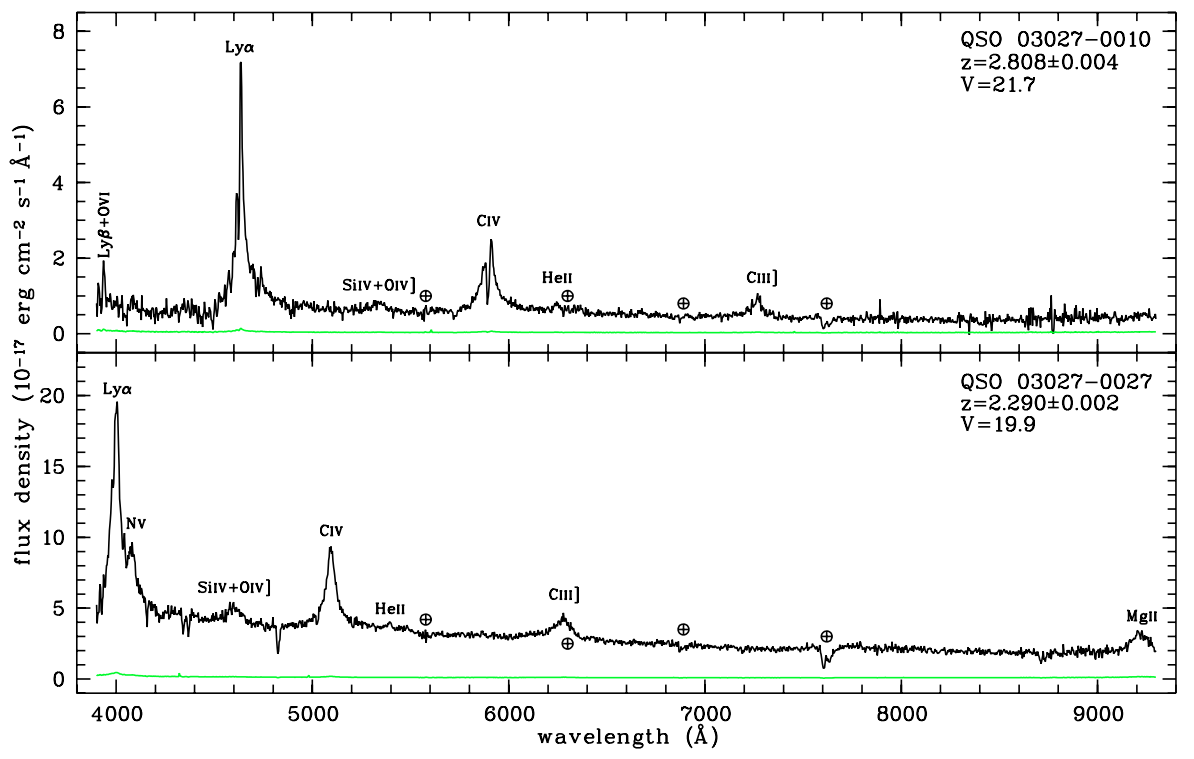

Fig. 2. VLT/FORS2 spectra of the two discovered QSOs. The spectra are shown in black lines together with their corresponding $1 \sigma$ noise arrays (green lines). Detected emission lines and major atmospheric artifacts are indicated.
Table 2. Redshift determination of the two newly discovered QSOs.

\begin{tabular}{lcccc}
\hline \hline Emission & \multicolumn{2}{c}{ QSO $03027-0010$} & \multicolumn{2}{c}{ QSO 03027-0027 } \\
line & $\lambda[\AA]$ & $z$ & $\lambda[\AA]$ & $z$ \\
\hline Ly $\beta+$ O VI & $3936 \pm 5$ & $2.810 \pm 0.005$ & - & - \\
Ly $\alpha$ & $4627 \pm 7$ & $2.806 \pm 0.006$ & $4004 \pm 3$ & $2.294 \pm 0.003$ \\
N v & - & - & $4081 \pm 3$ & $2.291 \pm 0.003$ \\
Si IV+O IV] & $5335 \pm 10$ & $2.812 \pm 0.007$ & $4598 \pm 8$ & $2.284 \pm 0.006$ \\
C IV & $5896 \pm 11$ & $2.806 \pm 0.007$ & $5095 \pm 2$ & $2.289 \pm 0.001$ \\
He II & $6244 \pm 3$ & $2.806 \pm 0.002$ & $5393 \pm 3$ & $2.288 \pm 0.002$ \\
C III $]$ & $7268 \pm 4$ & $2.808 \pm 0.002$ & $6278 \pm 5$ & $2.289 \pm 0.003$ \\
Mg II & - & - & $9223 \pm 15$ & $2.296 \pm 0.005$ \\
\hline
\end{tabular}

prevents a clear detection of low-ionization lines, such as $\mathrm{O} I+\mathrm{Si}$ II or $\mathrm{O} \mathrm{III}]$. The redshift measurement based on the Mg II line in QSO 03027-0027 is inaccurate due to the decreasing resolving power of the grism at the longest recordable wavelengths. Consequently, the redshifts have to be measured in high-ionization lines which suffer from systematic blueshifts (Gaskell 1982; Tytler \& Fan 1992; McIntosh et al. 1999). The measurements in the spectrum of QSO 03027-0010 are further affected by the associated absorption system. Having these caveats in mind, we adopt a redshift of $z=2.290 \pm 0.002$ for QSO 03027-0027 and a redshift of $z=2.808 \pm 0.004$ for QSO 03027-0010.

Apparent magnitudes of both quasars were derived based on the available spectrophotometry, yielding $V=21.7$ for QSO 03027-0010 and $V=19.9$ for QSO 03027-0027.

\subsection{Continuum and redshift of $Q 0302-003$}

In order to be able to use the FORS2 spectrum of Q 0302-003 for measuring its transmission properties in the $\mathrm{HI}$ Ly $\alpha$ forest region, we had to obtain a working estimate of the continuum. This was established by fitting a cubic spline to selected high-transmission regions of the data, using the DIPSO software under Starlink ${ }^{1}$. Due to the low resolution of the spectrum, the absorption lines are heavily blended. We checked our continuum fit for systematic errors using line lists from highresolution spectra published by $\mathrm{Hu}$ et al. (1995) from Keck HIRES data and by Kim et al. (2002) from VLT UVES data. The list from Hu et al. (1995) covers the redshift range $2.627 \leq$ $z \leq 3.110$, whereas the list from Kim et al. (2002) extends between $2.957 \leq z \leq 3.235$. The two line lists were used to create artificial Ly $\alpha$ forest spectra which were subsequently convolved with the line spread function of the FORS2 600B grism and rebinned to $1.5 \AA /$ pixel. The result was an excellent correspondence (rms error $\sim 3 \%$ ) between our data and the simulated spectra, indicating that our fit did not grossly underestimate the true continuum. The only regions where our continuum estimate is significantly too low by $\sim 8 \%$ are the wavelength range largely devoid of Ly $\alpha$ absorption from $\sim 5050 \AA$ to $\sim 5100 \AA$, known as the Dobrzycki \& Bechtold void (Dobrzycki \& Bechtold 1991a) and a small region at $\sim 4480 \AA$ already in the line wing of the $\mathrm{Ly} \beta+\mathrm{O}$ VI line of Q 0302-003 where continuum fitting becomes complicated.

1 The authors acknowledge the data analysis facilities provided by the Starlink Project which is run by CCLRC on behalf of PPARC. 
Table 3. Known $z>2$ foreground quasars within $30^{\prime}$ of Q $0302-003(z=3.285)$. We list positions, redshifts, $V$ magnitudes, projected angular distances $\vartheta$ from Q 0302-003, and the corresponding transverse proper distances $d_{\perp}(z)$ at the emission epoch of each foreground quasar.

\begin{tabular}{|c|c|c|c|c|c|c|c|c|}
\hline QSO & Abbr. & $\alpha(\mathrm{J} 2000)$ & $\delta(\mathrm{J} 2000)$ & $z$ & $V$ & $\vartheta\left[{ }^{\prime}\right]$ & $d_{\perp}[\mathrm{Mpc}]$ & discovery paper \\
\hline QSO 03022-0023 & $\mathrm{F}$ & $03^{\mathrm{h}} 04^{\mathrm{m}} 45^{\mathrm{s}} .94$ & $-00^{\circ} 11^{\prime} 38^{\prime \prime} .2$ & 2.142 & 22.5 & 3.55 & 1.77 & Jakobsen et al. (2003) \\
\hline QSO 03027-0027 & $\mathrm{E}$ & $03^{\mathrm{h}} 05^{\mathrm{m}} 15^{\mathrm{s}} .62$ & $-00^{\circ} 16^{\prime} 14^{\prime \prime} .4$ & 2.290 & 19.9 & 10.31 & 5.08 & this paper \\
\hline QSO 03027-0010 & $\mathrm{B}$ & $03^{\mathrm{h}} 05^{\mathrm{m}} 16^{\mathrm{s}} .80$ & $+00^{\circ} 00^{\prime} 45^{\prime \prime} .1$ & 2.808 & 21.7 & 11.24 & 5.29 & this paper \\
\hline Q $0302-D 113$ & $\mathrm{D}$ & $03^{\mathrm{h}} 04^{\mathrm{m}} 30^{\mathrm{s}} .33$ & $-00^{\circ} 08^{\prime} 11^{\prime \prime} .4$ & 2.920 & 24.3 & 4.85 & 2.26 & Steidel et al. (2003) \\
\hline QSO 03020-0014 & A & $03^{\mathrm{h}} 04^{\mathrm{m}} 35^{\mathrm{s}} .37$ & $-00^{\circ} 02^{\prime} 50 .^{\prime} 9$ & 3.050 & 20.5 & 6.46 & 2.97 & Jakobsen et al. (2003) \\
\hline Q 0301-005 & $\mathrm{C}$ & $03^{\mathrm{h}} 03^{\mathrm{m}} 41^{\mathrm{s}} .05$ & $-00^{\circ} 23^{\prime} 21^{\prime \prime} .8$ & 3.231 & 17.8 & 22.89 & 10.34 & Barbieri \& Cristiani (1986) \\
\hline
\end{tabular}

We also checked the line lists of Hu et al. (1995) and Kim et al. (2002) for metal line contamination of the H I Ly $\alpha$ forest. Only a few identified systems contaminate the Ly $\alpha$ forest. These are narrow lines with low column densities, which are heavily blended with $\mathrm{HI}$ lines at our low resolution. The absorption by metals is therefore negligible and we will treat the whole absorption in the spectral range of the $\mathrm{H}$ I Ly $\alpha$ forest as genuine H I Ly $\alpha$ absorption.

Our FORS2 spectrum of Q 0302-003 covers the wavelength range from the UV cutoff to $\sim 6350 \AA$ and has a $S / N$ of $\sim 75$ in the quasar continuum, so we can measure the positions of the lines Ly $\beta+\mathrm{O}$ VI, Ly $\alpha, \mathrm{N} \mathrm{V}, \mathrm{O}$ I+Si II, C II and $\mathrm{Si}$ IV+OIV]. We base our redshift measurement on the lowionization lines $\mathrm{O}$ I+Si II and $\mathrm{C}$ II, which should yield the best possible estimate of the systemic redshift. We get a redshift of $z=3.285 \pm 0.001$ for the O I+Si II line and a redshift of

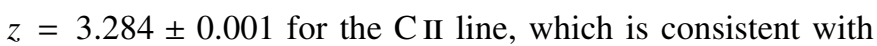
the value of 3.286 originally obtained by Sargent et al. (1989). But we note that their value was based on the Ly $\alpha$ and the C IV emission line.

The sky region around Q 0302-003 was targeted by SDSS, and Q 0302-003 was observed spectroscopically. We checked the SDSS DR3 database for the spectroscopic redshift of Q $0302-003$ and found the redshift to be too high, $z_{\text {SDSS }} \simeq$ $3.295 \pm 0.001$. Given this large offset of $\Delta z=0.009$, we obtained the SDSS spectrum of Q 0302-003 from the SDSS data archive and recovered the redshift values for the low-ionization lines $\mathrm{O}$ I+Si II $(z=3.286 \pm 0.002)$ and C II $(z=3.284 \pm$ $0.002)$. We conclude that the SDSS redshift assignment for Q $0302-003$ is incorrect and adopt a redshift of $z=3.285$ for this quasar in the following.

\subsection{HST/STIS data}

Q 0302-003 was one of the few high-redshift quasars observed successfully in the He II Ly $\alpha$ forest below $303.78 \AA$ rest frame wavelength with HST and its Space Telescope Imaging Spectrograph at a resolution of $1.8 \AA$ (Heap et al. 2000, hereafter refered to as $\mathrm{H} 00$ ). We retrieved these data from the HST archive and re-reduced them using CALSTIS v2.13 distributed with IRAF. As in the reduction by $\mathrm{H} 00$, we adjusted the background subtraction windows in order to correct for the spatial variability of the STIS MAMA1 detector background. We adopt their flux normalization with a power law $f_{v} \propto v^{-\alpha}$ with $\alpha=2$, yielding $f_{\lambda}=$ const. $\simeq 2.1 \times 10^{-16} \mathrm{erg} \mathrm{cm}^{-2} \mathrm{~s}^{-1} \AA^{-1}$.

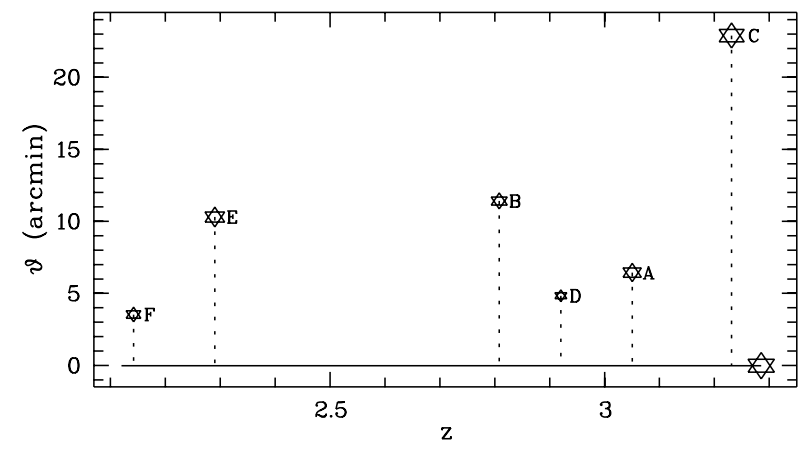

Fig. 3. Distribution of the foreground quasars from Table 3 with respect to Q 0302-003. Symbol size indicates apparent optical magnitude.

The spectral index of 2 is consistent with the composite QSO EUV spectral index of $\sim 1.8$ by Telfer et al. (2002).

\subsection{Quasars in the vicinity of $Q 0302-003$}

Table 3 tabulates all 6 known quasars at $z>2$ within a radius $<30^{\prime}$ around Q 0302-003. Their distribution in redshift and separation from the central line of sight is shown in Fig. 3. In addition to the QSOs discovered or rediscovered in our survey, we found two further quasars listed by Véron-Cetty \& Véron (2003). Spectra for these QSOs were retrieved from public archives, in order to measure the emission redshifts as consistently as possible. For the sake of clarity, we will use a simplified nomenclature in the following and denote the foreground QSOs by the letters A ...F as indicated in Table 3.

Q 0301-005 (QSO C) is a bright QSO known since long. It was observed by SDSS and we obtained its spectrum from the SDSS data archive. Previous redshift determinations were based on high-ionization lines (Sargent et al. 1989), so its redshift of $z=3.223$ measured by Sargent et al. (1989) may have been underestimated. The SDSS spectrum of this QSO has sufficient $S / N$ to measure the low-ionization lines O I+Si II and C II, yielding a redshift of $z=3.231$ for this object in agreement with the SDSS redshift assignment. Note the large shift of $\Delta z=0.008$ with respect to the measurement based on highionization lines.

the two quasars discovered by Jakobsen et al. (2003, hereafter refered to as J03) have spectra taken with FORS1 in the ESO/VLT Science Archive. We performed an independent 


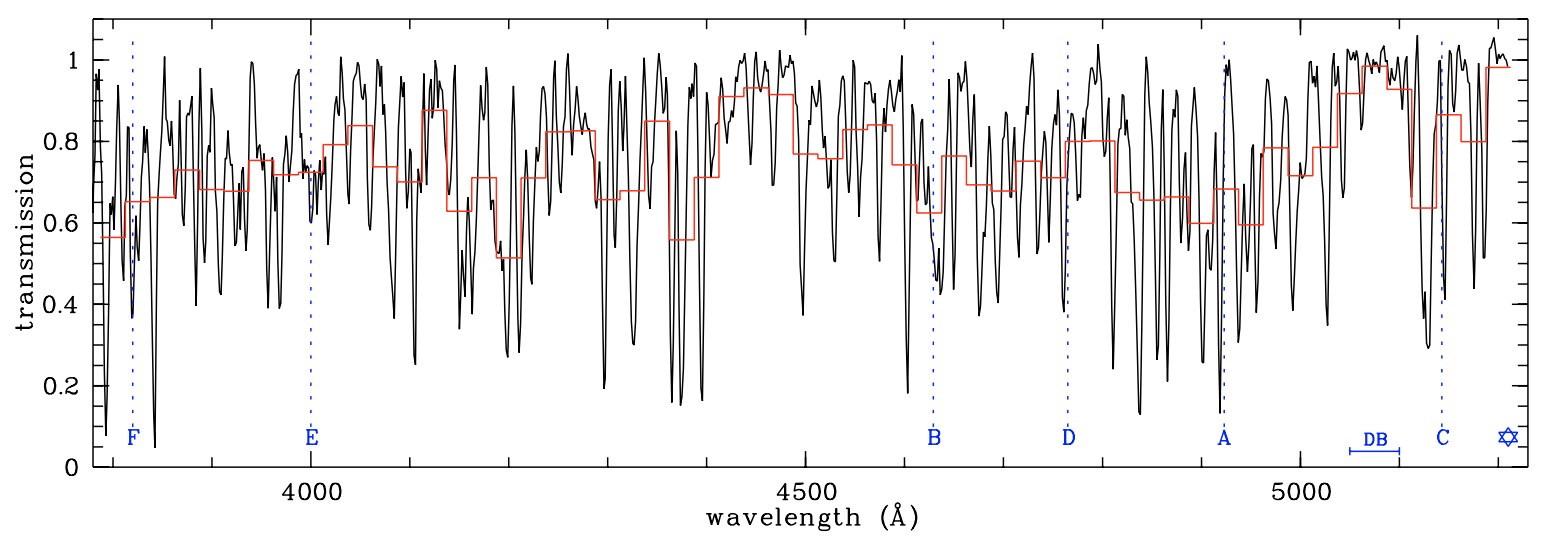

Fig. 4. Transmission spectrum of the $\mathrm{H}$ I Ly $\alpha$ \& Ly $\beta$ forest of Q 0302-003. The spectrum is shown in black from $3780 \AA$ to $5210 \AA$ (corresponding to the Ly $\alpha$ emission peak of Q 0302-003). Blue letters and vertical dotted lines mark the foreground quasars from Table 3 projected into the forest. We also indicated the background quasar Q 0302-003 by a star symbol and the void identified by Dobrzycki \& Bechtold (1991a) (DB). The binned red line is the average transmission in $25 \AA$ bins. [See the online edition of the Journal for a colour version of this figure.]

reduction and give here a refined redshift (redshift uncertainty 0.002) and the magnitude for QSO 03022-0023 (QSO F). With $V=22.5$ it is too faint to be detected in our slitless spectroscopic survey material. Our estimate of redshift and redshift error for QSO 03020-0014 (QSO A) agrees with the values given in $\mathrm{J} 03$.

Q 0302-D113 (QSO D) was published by Steidel et al. (2003) after the compilation of the Véron-Cetty \& Véron (2003) catalog. No spectrum is available for this very faint $(R \simeq 24.6)$ object. Its $V$ magnitude in Table 3 is estimated from $R$.

\section{No visible $\mathrm{HI}$ transverse proximity effect}

We first conducted a simple visual search for locally enhanced transmission of the H I Lyman forest at the location of the foreground quasars. Figure 4 displays our low-resolution transmission spectrum of Q 0302-003, together with the effective locations of the foreground quasars projected into the forest. The proximity effect manifests itself as a statistical increase in Ly $\alpha$ forest transmission near a quasar. Already a quick examination of Fig. 4 shows that there are at least no obvious H I voids located at any of the foreground quasars that could be revealed by visual inspection, with the possible exception of QSO A which is located very close to a local transmission maximum. There is no such correlation for any of the other QSOs. There is, however, a significant increase of Ly $\alpha$ forest transmission towards the Ly $\alpha$ line of the central QSO Q 0302-003 itself.

Considering the luminosities of the foreground QSOs and their projected angular distances from the central line of sight, this lack of a transverse proximity effect in $\mathrm{HI}$ is no real surprise. We computed the parameter

$$
\omega(z)=\sum_{j=1}^{n} \frac{f_{v_{\mathrm{LL}}, j}}{4 \pi J_{v}(z)} \frac{\left(1+z_{j}^{\prime}\right)^{-\alpha_{j}+1}}{\left(1+z_{j}\right)}\left(\frac{\alpha_{J_{v}}+3}{\alpha_{j}+3}\right)\left(\frac{d_{L}\left(z_{j}, 0\right)}{d_{L}\left(z_{j}, z\right)}\right)^{2}
$$

which is the ratio between the summed photoionization rates of $n$ quasars at redshifts $z_{j}$ with a rest frame Lyman limit
Table 4. Rest frame Lyman limit fluxes of Q 0302-003 and all nearby QSOs. A power law $f_{v} \propto v^{-\alpha}$ is fitted to all quasars with an available spectrum. $f_{v_{\mathrm{LL}}}$ is the extrapolated Lyman limit flux in the QSO rest frame and $\omega\left(z_{\mathrm{em}}\right)$ is the resulting predicted strength of the transverse proximity effect (see text).

\begin{tabular}{lllll}
\hline \hline QSO & Abbr. & $\alpha$ & $f_{v_{\mathrm{LL}}}[\mu \mathrm{Jy}]$ & $\omega\left(z_{\mathrm{em}}\right)$ \\
\hline QSO 03022-0023 & F & $1.12 \pm 0.06$ & $2 \pm 4$ & 0.073 \\
QSO 03027-0027 & E & $0.67 \pm 0.02$ & $22 \pm 17$ & 0.121 \\
QSO 03027-0010 & B & $0.88 \pm 0.02$ & $4 \pm 4$ & 0.035 \\
QSO 0302-D113 & D & & $0.3 \pm 0.3$ & 0.028 \\
QSO 03020-0014 & A & $1.42 \pm 0.01$ & $10 \pm 7$ & 0.253 \\
Q 0301-005 & C & $0.90 \pm 0.01$ & $136 \pm 89$ & 0.776 \\
Q 0302-003 & $\star$ & $0.50 \pm 0.01$ & $222 \pm 124$ & $\infty$ \\
\hline
\end{tabular}

flux $f_{v_{\mathrm{LL}}, j}$, penetrating the absorber at redshift $z$ and the overall UV background with Lyman-limit intensity $J_{v} . d_{L}\left(z_{j}, 0\right)$ is the luminosity distance of QSO $j$, and $d_{L}\left(z_{j}, z\right)$ is its luminosity distance as seen at the absorber; the redshift of the quasar as seen at the absorber is $z_{j}^{\prime}$ (Liske 2000). Thus, $\omega\left(z_{\mathrm{em}}\right)$ characterizes the expected strength of the transverse proximity effect signature at a given QSO redshift along the line of sight: A highly significant effect would require $\omega\left(z_{\mathrm{em}}\right) \gg 1$, i.e. a UV radiation field dominated by the local source.

We fixed the UV background at 1 ryd to $J_{v}=7 \times$ $10^{-22} \mathrm{erg} \mathrm{cm}^{-2} \mathrm{~s}^{-1} \mathrm{~Hz}^{-1} \mathrm{sr}^{-1}$ (Scott et al. 2000) and assumed it to be constant over the relevant redshift range $2<z<3.285$ with a power-law shape $J_{v} \propto v^{-\alpha_{J_{v}}}$ and $\alpha_{J_{v}}=1.5$. The quasar Lyman limit fluxes were estimated from the available spectra by fitting a power law $f_{v} \propto v^{-\alpha}$ to the quasar continuum redward of the Ly $\alpha$ emission line, excluding the emission lines. Table 4 lists the resulting spectral indices and the Lyman limit fluxes for the quasars. For QSO D there was no spectrum available and we estimated its Lyman limit flux from its $R$ magnitude assuming a power law index $\alpha=0.5$.

In all cases except for Q 0302-003 itself we find peak $\omega$ values significantly below unity. The highest value is 


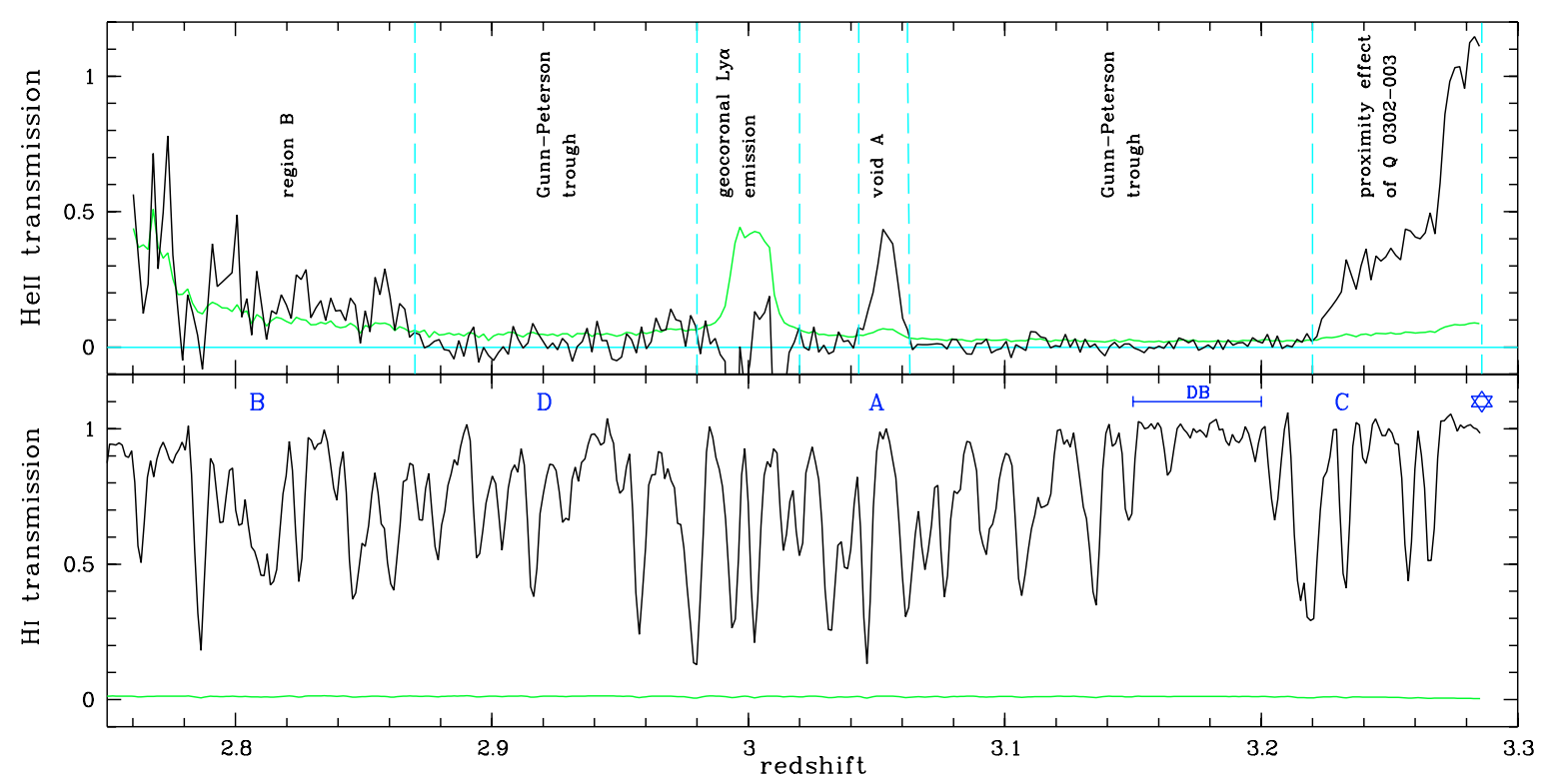

Fig. 5. Comparison of the He II and the H I Ly $\alpha$ absorption spectrum towards Q 0302-003. Upper panel: transmission in He II Ly $\alpha$ vs. redshift from the HST/STIS data obtained by H00. The green curve shows the $1 \sigma$ uncertainty per pixel. The horizontal cyan line denotes the zero flux level. Vertical dashed lines divide the spectrum into several labeled regions of interest (see text). The lower panel displays the corresponding section of our FORS2 H I Ly $\alpha$ transmission spectrum. Quasar and void locations are marked as in Fig. 4. [See the online edition of the Journal for a colour version of this figure.]

reached by the very luminous QSO C, for which Dobrzycki $\&$ Bechtold (1991a) proposed that it might cause the large void at $z \sim 3.17$. While the redshift offset between this void and QSO C at $z=3.231$ may still be explained by anisotropic emission, the small $\omega\left(z_{\mathrm{em}}\right)$ value implies that this quasar would have to be implausibly bright in the direction of the void (Dobrzycki \& Bechtold 1991b). Note that due to their small redshift difference, Q 0302-003 and QSO C have similar impact at $z \simeq 3.231$. The $\omega\left(z_{\mathrm{em}}\right)$ value of QSO C is therefore the sum of the contributions from these two sources. The small projected proximity effect zone of QSO C merges with the onset of the much larger line of sight proximity effect zone caused by Q 0302-003 itself. The refined redshift of QSO C implies an even larger offset between quasar and void; together with the modest value of $\omega$ it follows that the Dobrzycki \& Bechtold (1991a) void is very unlikely related to the ionizing radiation from QSO C. This statement will be reinforced below in Sect. 5.

For all other QSOs the $\omega$ values are even much smaller. Consequently, as long as one assumes roughly isotropic radiation, these QSOs should leave no individually detectable traces of a transverse proximity effect. This also holds for QSO A. Its matching redshift with a small $\mathrm{HI}$ void could therefore be just a coincidence, especially when considering the large number of similar features in the spectrum. Nevertheless, the combined evidence from $\mathrm{HI}$ and He II forests suggests that this region does indeed receive a substantial amount of hard QSO radiation (H00, J03).

\section{Fluctuations in the He II Lyman forest}

Now we turn to the He II Ly $\alpha$ absorption observed with STIS on HST in the redshift range $2.76<z<3.285$ by H00. Figure 5 compares the transmission spectra of the He II and H I Ly $\alpha$ absorption towards Q 0302-003 as a function of redshift. The main features are labeled following $\mathrm{H} 00$.

There are only three confined regions of significantly nonzero transmission in the He II spectrum. Firstly, the redshift range from $z=3.22$ up to the emission redshift of Q 0302-003 of $z_{\mathrm{em}}=3.285$ displays strongly enhanced transparency, presumably through a strong line of sight proximity effect created by a Strömgren sphere in an IGM where He II is still not reionized to He III (Hogan et al. 1997, H00). The luminous QSO C is located within the extent of this zone, albeit near its lowredshift end.

Second, there is a prominent transmission feature at $3.043<z<3.063$ ("void A"). H00 speculated that this was likely to be created by a nearby AGN. J03 found the closely coinciding quasar QSO 03020-0014 (QSO A) and argued that this QSO most likely was responsible for the void, although they invoked some modest degree of anisotropic radiation in order to explain an apparent shift between the QSO at $z=$ $3.050 \pm 0.003$ and their stated peak of void $\mathrm{A}$ at $z=3.056$. From our measurement the peak of void A is at redshift $z=3.052$ which is perfectly consistent with the QSO being located symmetric to the void.

The third transmission window is a considerably wider region at $z \lesssim 2.87$ ("region B"). The $S / N$ per pixel in the STIS data is low, but the transmission nearly continuously exceeds the noise. Region B is located at the low-redshift end of the STIS spectrum and the detector sensitivity strongly decreases towards the cutoff at $z \sim 2.76$, but it is likely that we see the onset of a proper He II Ly $\alpha$ forest. This interpretation is supported by the large extent of this region with inherent 
substructure and a spectral hardening of the radiation field in region $\mathrm{B}$ found by $\mathrm{H} 00$.

QSO B falls into the redshift range of region $\mathrm{B}$, but that region is much too broad to allow for a close connection between this quasar and the overall enhancement of transparency. However, we notice that while the H I forest near QSO B actually shows significant absorption there is no corresponding depression in the He II forest as would be expected for a roughly constant column density ratio. We tentatively conclude that this ratio may change significantly near QSO B, possibly indicating the radiative influence of the quasar.

\section{The spectral hardness of the UV radiation field}

\subsection{Diagnostics}

A key parameter to study the radiative influence of a given quasar on the IGM is the relative hardness of the UV radiation ionizing the intergalactic matter. There are several established ways to express this hardness. If the shape of the ionizing continuum is known, the spectral softness parameter is defined as the ratio between the ionization rates of $\mathrm{H}$ I and $\mathrm{He}$ II,

$S=\frac{\Gamma_{\mathrm{HI}}}{\Gamma_{\text {He II }}} \simeq 4^{\alpha+1}$

with $\alpha$ as the relevant spectral index, $f_{v} \propto v^{-\alpha}$. More indirectly, the hardness can be described by the ratio $\eta$ of the column densities of He II and $\mathrm{HI}$ at given $z$,

$\eta=\frac{N_{\mathrm{He} \text { II }}}{N_{\mathrm{HI}}}$

This quantity is theoretically predicted to be $\lesssim 100$ for a hard ionizing field with mostly quasars acting as ionizing sources, and $\gtrsim 100$ for a soft radiation field that is dominated by galaxies (Haardt \& Madau 1996; Fardal et al. 1998); typically, $S \simeq 2.3 \eta$ if $\mathrm{HI}$ and He II are highly ionized. Unfortunately it is hard to measure $\eta$ directly, because of the limited spectral resolution of the FUV spectrographs aboard HST. Only for the two lines of sight towards HE 2347-4342 and HS 1700+6416, FUSE spectra of sufficient resolution have allowed direct measurements of He II column densities and $\eta$ (Kriss et al. 2001; Zheng et al. 2004b; Shull et al. 2004; Reimers et al. 2004), revealing fluctuations of $\eta$ on very small scales of $\Delta z \approx 10^{-3}$, indicating the presence of hard and soft photoionizing sources whose radiation is filtered through the cosmic web.

At lower resolution a technique has been applied that allows one to at least roughly estimate $\eta$. Using accurately measured H I column densities from optical spectroscopy, one assumes a value for $\eta$, generates the ensuing He II spectrum, degrades it to the actual resolution of the UV spectrum and compares the simulation with the data (Reimers et al. 1997; Hogan et al. 1997; Anderson et al. 1999; Heap et al. 2000; Smette et al. 2002). These studies showed consistently and in agreement with the higher resolution FUSE results that $\eta$ significantly fluctuates over the entire redshift range covered by a given line of sight. On this basis $\mathrm{H} 00$ predicted that void A in Q 0302-003 had to be created by a QSO because only a small value of $\eta \sim 50$ could reproduce the STIS spectrum in this region, whereas outside of it $\eta$ was much larger. Subsequently J03 discovered QSO A almost exactly at the predicted redshift.

An alternative to the above procedure is given by evaluating the ratio $R$ of the effective optical depths of He II and H I,

$R \equiv \frac{\tau_{\text {eff,He II }}}{\tau_{\text {eff }, \mathrm{HI}}}$

which has the same overall characteristics as $\eta$, but is resolution-independent. High (small) values of $R$ will be obtained if the He II absorption is high (small) compared to $\mathrm{H} \mathrm{I}$, indicating a soft (hard) photoionizing field.

We now want to investigate fluctuations of $\eta$ in other parts of the Q 0302-003 spectrum, notably in region B. Our approach is therefore reverse to that of $\mathrm{HOO}$ and $\mathrm{J} 03$, in that we have now specific redshifts given by quasars, and we are interested in the behaviour of $\eta$ at these redshifts. However, we first need to consider the question of how much these results depend on unresolved weak Lyman forest lines.

\subsection{Unresolved weak $H$ I forest lines}

For given H I column density, the He II absorption is typically much stronger than the corresponding H I absorption ( $\eta \gg 1)$. He II can therefore be traced in absorbers with $\log N_{\mathrm{HI}} \lesssim 12$, where the more diffuse component of the IGM sets in - effectively a shallow Gunn-Peterson trough (e.g. Songaila et al. 1995; Fardal et al. 1998). This IGM component is very hard to account for in the HI forest because its detection relies on small shallow excursions of the data from the assumed quasar continuum that can easily get lost in the fitting process. Almost every quasar continuum in high-resolution data is defined locally, so one typically does not account for this low-density IGM component. This poses a fundamental problem when trying to estimate the spectral hardness of the UV radiation field by comparing the absorption in H I and He II. The derived values for $\eta$ and $R$ will be systematically overestimated when the true $\mathrm{H}$ I absorption is underestimated.

The best approach to account for the additional undetected $\mathrm{HI}$ absorption is by resolving the He II absorption into discrete lines and using the He II forest to predict the low end of the H I column density distribution (Kriss et al. 2001; Zheng et al. $2004 b$ ). This option is available only for two quasar lines of sight with FUSE data.

Other possibilities are to use analytic approximations to calculate the mean Ly $\alpha$ forest absorption arising from these lines (Møller \& Jakobsen 1990; Zuo 1993; Madau 1995) or to simulate their impact with empirically established distribution laws. Noting that the line lists of $\mathrm{Hu}$ et al. (1995) and Kim et al. (2002) become grossly incomplete below $\log N_{\mathrm{H}} \simeq 12$, we estimated the contribution of $9 \leq \log N_{\mathrm{HI}} \leq 12$ systems to the total absorption. This yields a lower limit on $\tau_{\mathrm{GP}}$ from completely undetected absorption lines. We assumed a power law-shape column density distribution function with slope $\beta=1.5$ and used the empirical redshift distribution parameterization from Kim et al. (2002). From both simulation and analytic approximation we obtained an effective optical depth of $\tau_{\text {eff }} \simeq 0.02$ for this column density range. 
A suitable correction has to be higher than this lower limit due to the partial incompleteness of the line lists at $\log N_{\mathrm{H} \text { I }} \lesssim$ 13. $\mathrm{H} 00$ generated weak forest lines with $9 \leq \log N_{\mathrm{HI}} \leq 13$ by extrapolating the distribution functions from Kim et al. (1997), resulting in an estimate of $\tau_{\mathrm{GP}} \simeq 0.06$. Our best-guess value is somewhat lower than this; based on exploring various parameterizations of the distribution functions for the range $12 \leq \log N_{\mathrm{HI}} \leq 13$, we adopted an overall $\tau_{\mathrm{GP}} \simeq 0.04$.

We applied this correction over the whole redshift range of interest by multipying our FORS2 transmission spectrum by a factor $\left\langle T_{\mathrm{H}, \mathrm{GP}}\right\rangle=0.96$. The main source of uncertainty of both used approaches lies certainly in the extrapolation of the column density distribution to below the validated range. However, this is not a major problem for our study because we are primarily interested in spatial fluctuations of $R$ and $\eta$ rather than absolute values.

The additional absorption in $\operatorname{He}$ II $\operatorname{Ly} \alpha$ that arises from the cloud population not observed in H I can be easily calculated. These absorbers are optically thin both in H I and He II, so that

$\left\langle T_{\mathrm{He} \text { II }, \mathrm{GP}}\right\rangle=\left\langle T_{\mathrm{HI}, \mathrm{GP}}\right\rangle^{\eta / 4}$.

Applying the H I transmission correction factor gives a global He II transmission correction as a function of $\eta$. Simulated He II spectra created from incomplete H I line lists have to be multiplied by $\left\langle T_{\mathrm{He} \text { II,GP }}\right\rangle$. Since the depth of the shallow H I Gunn-Peterson trough is not exactly known, a degeneracy in the hardness parameter $\eta$ arises due to the strong dependence of the $\eta$ value fitting the data on the assumed $\left\langle T_{\mathrm{H}, \mathrm{GP}}\right\rangle$. From Fig. 6 we find that all simulated He II spectra except those with very low $(\eta \simeq 1)$ or very high assumed $\eta$ depend strongly on the value of $\left\langle T_{\mathrm{H} \text { I,GP }}\right\rangle$. Any study based on low-resolution He II spectra will therefore be plagued by the ambiguity that different $\eta$ values will fit the data, depending on the adopted correction for low-column density material. The best-fitting $\eta$ will overestimate (underestimate) the true value if $\left\langle T_{\mathrm{H}, \mathrm{GP}}\right\rangle$ is set too high (low). As a consequence of this degeneracy, the spectral hardness values derived from low-resolution He II data have an uncertain absolute scale. However, any indications for fluctuations in the fitting $\eta$ values along the line of sight due to a changing hardness of the ionizing field will remain valid, unless the shallow Gunn-Peterson trough correction itself fluctuates with a similar rate.

\subsection{The fluctuating optical depth ratio}

In order to apply the above defined hardness indicator $R$, we binned the STIS spectrum and the FORS2 spectrum of Q 0302-003 into aligned redshift bins of common size. For the STIS spectrum we adopted the wavelength resolution of $\Delta \lambda \simeq 1.8 \AA$ as estimated by $\mathrm{H} 00$ and binned both transmission spectra into $\Delta z=0.006$ bins starting at $z=2.76$ up to the emission redshift of Q 0302-003 at $z=3.285$. This rebinning implies that one has to deal with original flux bins that only partly overlap with the new bins. We adopted the method from Telfer et al. (2002), who weighted each original flux by the extent of the overlap with the new bin. The errors were computed accordingly.

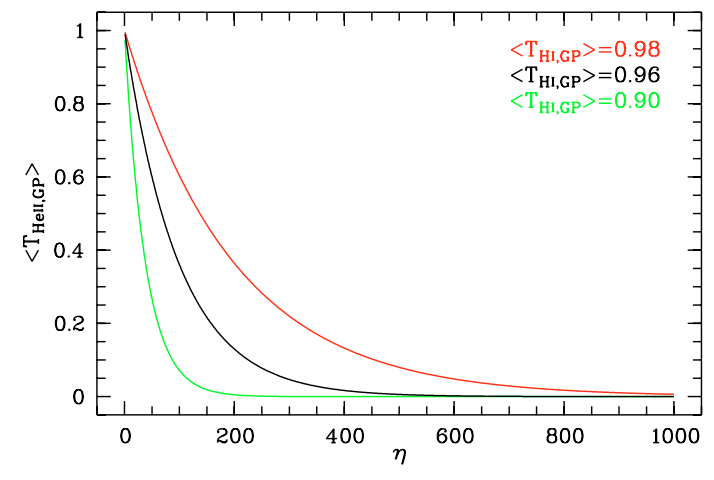

Fig. 6. $\left\langle T_{\mathrm{He} \text { II,GP }}\right\rangle$ vs. $\eta$ for different $\left\langle T_{\mathrm{H}, \mathrm{GP}}\right\rangle$. In the range of physical $\eta$ values, the He II transmission correction is strongly dependent on $\left\langle T_{\mathrm{HI}, \mathrm{GP}}\right\rangle$. [See the online edition of the Journal for a colour version of this figure.]

The optical depth ratio $R=\ln \left\langle T_{\mathrm{He} \text { II }}\right\rangle / \ln \left\langle T_{\mathrm{HI}_{\mathrm{I}}}\right\rangle$ would obtain unphysical values if $\langle T\rangle \leq 0$ or $\langle T\rangle \geq 1$. In our case only the He II spectrum is affected. Two $\left\langle T_{\text {He II }}\right\rangle \geq 1$ bins near Q 0302-003 are contaminated by its blue He II emission line wing. Due to their location in the proximity effect zone, we estimate $\left\langle T_{\text {He II }}\right\rangle \gtrsim 0.9$, corresponding to upper limits on $R$. Values with $\left\langle T_{\text {He II }}\right\rangle \leq 0$ have been replaced by their errors, yielding upper limits on $\left\langle T_{\mathrm{He}} \mathrm{II}\right\rangle$ and lower limits on $R$. The region $2.98<z<3.02$ is excluded because of the contamination from the geocoronal $\operatorname{Ly} \alpha$ emission line in the STIS spectrum. Error bars for $R$ were computed by propagating the transmission errors.

The result for all created redshift bins is shown in Fig. 7. One clearly sees a large variation in $R$, ranging from values of a few to $\gtrsim 50$, indicating substantial spectral fluctuations in the UV radiation field. Most interestingly, $R$ reaches a local minimum near every quasar along the line of sight, as expected for hard UV sources. Specifically, $R \simeq 3$ near all known quasars in the field except maybe QSO D, for which we have only a single bin at $z=2.916$ with a small value of $R \simeq 4$. Notice that $R \simeq 3$ also close to Q 0302-003 itself, and again so at the redshift of QSO C.

In order to estimate the statistical significance to find a local $R \lesssim 3$ minimum near the foreground quasars we performed Monte Carlo simulations. By randomly distributing quasars over the considered redshift range we estimate from 10000 simulations a probability of $\sim 9.3 \%$ that a single quasar falls into a redshift bin that corresponds to a local $R$ minimum at $\log (R)<0.5$. Since three foreground quasars (QSO A, B and $\mathrm{C}$ ) fall in such a minimum, the probability to find this constellation by chance is $<0.1 \%$.

Figure 7 shows also that the radiation field in the Dobrzycki \& Bechtold (1991a) H I void appears to be very soft, as already observed by H00. The errors are substantial, however, because throughout the void there is still a Gunn-Peterson trough seen in He II, with only very weak absorption in H I. There is a clear trend of higher $R$ values when approaching the approximate center of the void at $z \simeq 3.175$, reaching up to $R \sim 200$.

Finally we note that in Fig. 7 there are three narrow redshift regions at $z=2.778, z=2.832$ and $z=2.940$ where the local radiation field seems to be quite soft with $R \gtrsim 20$. From Fig. 5 


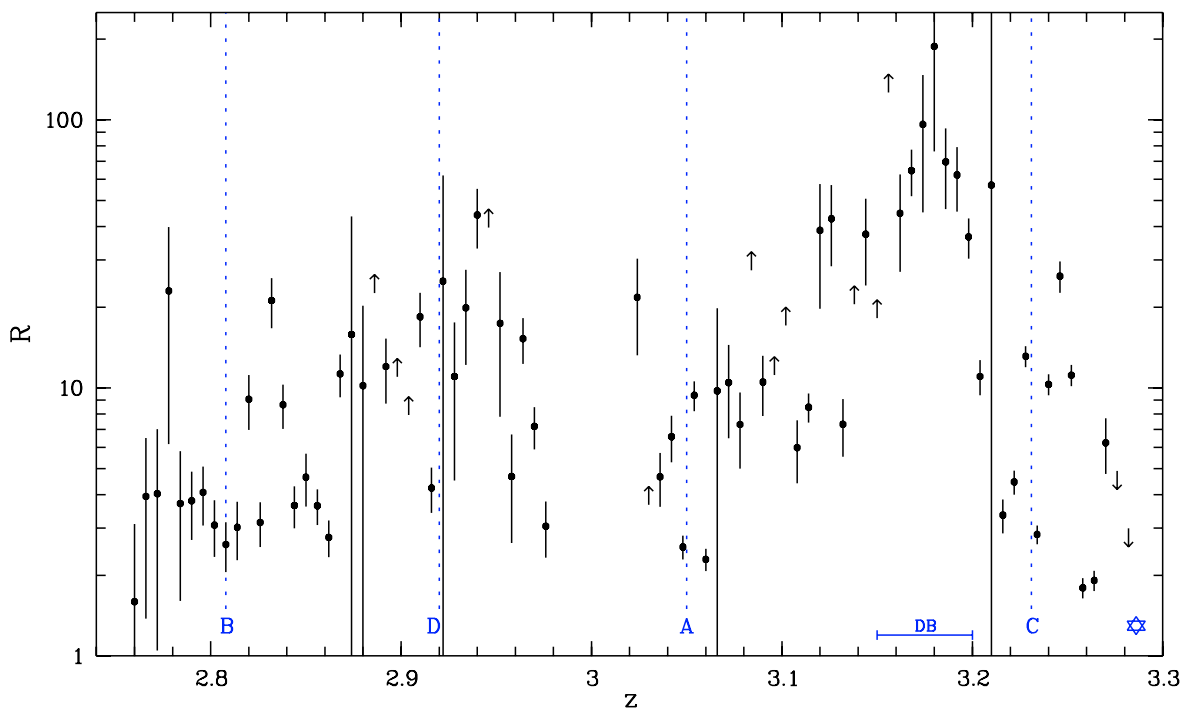

Fig. 7. Ratio of effective optical depths $R$ vs. redshift $z$. Arrows indicate upper and lower limits. Quasar and void locations are marked as in Fig. 4. Note that $R$ reaches small values near every quasar along the line of sight. There is a distinctive local minimum in $R$ at the redshift of QSO B. [See the online edition of the Journal for a colour version of this figure.] we see that these high $R$ values correspond to small $\mathrm{H}$ I voids. Such a correlation has been observed already in the highresolution studies with FUSE towards HE 2347-4342 (Kriss et al. 2001; Shull et al. 2004) and may indicate the contribution of star-forming galaxies to the UV background or quasar radiation that has been filtered and softened by radiative transfer through the cosmic web. The HST data for Q 0302-003 are insufficient to assess this in any detail.

\subsection{The fluctuating column density ratio}

We now turn to the more indirect approach of estimating $\eta$ through comparing the predicted with the observed He II absorption. We used the H I line lists of Hu et al. (1995) and Kim et al. (2002), which jointly cover the redshift range accessible with STIS in He II Ly $\alpha$. The Hu et al. (1995) list covers the range $2.627 \leq z \leq 3.110$, where the QSOs $\mathrm{B}, \mathrm{D}$, and $\mathrm{A}$ are located, whereas the list from Kim et al. (2002) extends between $2.957 \leq z \leq 3.235$, including QSOs $\mathrm{A}$ and $\mathrm{C}$, the $\mathrm{HI}$ void by Dobrzycki \& Bechtold (1991a). Only a small part of the line of sight proximity effect zone of Q 0302-003 itself is covered.

We simulated the Voigt profiles of the He II Ly $\alpha$ transition by assuming pure non-thermal broadening of the lines $\left(b_{\mathrm{He} \text { II }}=b_{\mathrm{HI}}\right)$ and a constant value for $\eta$ along the line of sight that was used to convert the observed $N_{\mathrm{H} \text { I }}$ into $N_{\mathrm{He} \text { II }}$. Non-thermal broadening found in simulations can be either due to turbulent motions of the gas or the differential Hubble flow, with the latter being dominant for the low-column density forest (Zhang et al. 1995, 1998; Hernquist et al. 1996; Weinberg et al. 1997; Bryan et al. 1999). Additionally Zheng et al. (2004b) presented observational evidence for $b_{\mathrm{He} \text { II }} \simeq b_{\mathrm{HI}}$. We then applied the He II transmission correction factor for undetected material in $\mathrm{HI}\left\langle T_{\mathrm{He} \text { II,GP }}\right\rangle \simeq 0.96^{\eta / 4}$. We performed the simulations for a range of $\eta$ values from 15 to 3000 . Finally, we degraded the resolution of the He II transmission spectrum to the actual STIS resolution by convolving it with a Gaussian line spread function of $1.8 \AA F W H M$ and rebinning the spectrum to the STIS pixel size of $0.6 \AA$.
The results for four representative values of $\eta$ are shown in Fig. 8 as the difference between the observed and the simulated spectrum, where positive (negative) deviations from zero indicate that $\eta$ has to be smaller (higher) than the assumed $\eta$ of the curve. One can clearly see that different values of $\eta$ match to different redshift regions, so the hardness of the ionizing radiation field has to fluctuate along the line of sight. Remarkably, the spectral regions around most of the quasars are best reproduced by low $\eta$ values, which are typical of quasars (Haardt \& Madau 1996; Fardal et al. 1998).

The onset of the line-of-sight proximity effect region of Q 0302-003 is reproduced with $\eta \sim 50$. H00 analyzed the proximity effect zone of Q 0302-003 in detail and found that $\eta$ strongly decreases when approaching Q 0302-003. Thus, in the proximity effect zone of Q $0302-003$ hard radiation is present that is softened with increasing distance to the quasar.

In the nearby H I void found by Dobrzycki \& Bechtold (1991a), a physical underdensity or a very soft radiation field with $\eta \gtrsim 350$ is required, as already noted by H00. Such high $\eta$ values are inconsistent with quasars as main ionizing sources. Furthermore, neither the survey by J03 nor our survey did reveal previously unknown quasars close to the void. As discussed in Sect. 3 above, QSO C is presumably not capable of creating the void. There is also no large overdensity of LBGs observed in the redshift range of the void that might photoionize the H I (Adelberger et al. 2003). Most likely, this void is a large-scale structure feature and not one created by local photoionizing sources.

J03 associated QSO A with void A mainly by showing that such an association has a very small probability of chance occurrence, and that the luminosity of QSO A is high enough to account for the void. Figure 8 shows clearly that most parts of void A can be fitted with $25 \lesssim \eta \lesssim 50$, which clearly favours a transverse proximity effect from a quasar.

Region B is quite crowded, and in Fig. 9 we present an enlarged view of Fig. 8 for this redshift range. The best-fit value of $\eta$ fluctuates between $\eta \sim 100$ and $\sim 25$ on scales comparable to the spectral resolution of the STIS data. This overall hardening of the metagalactic UV radiation field compared to the 


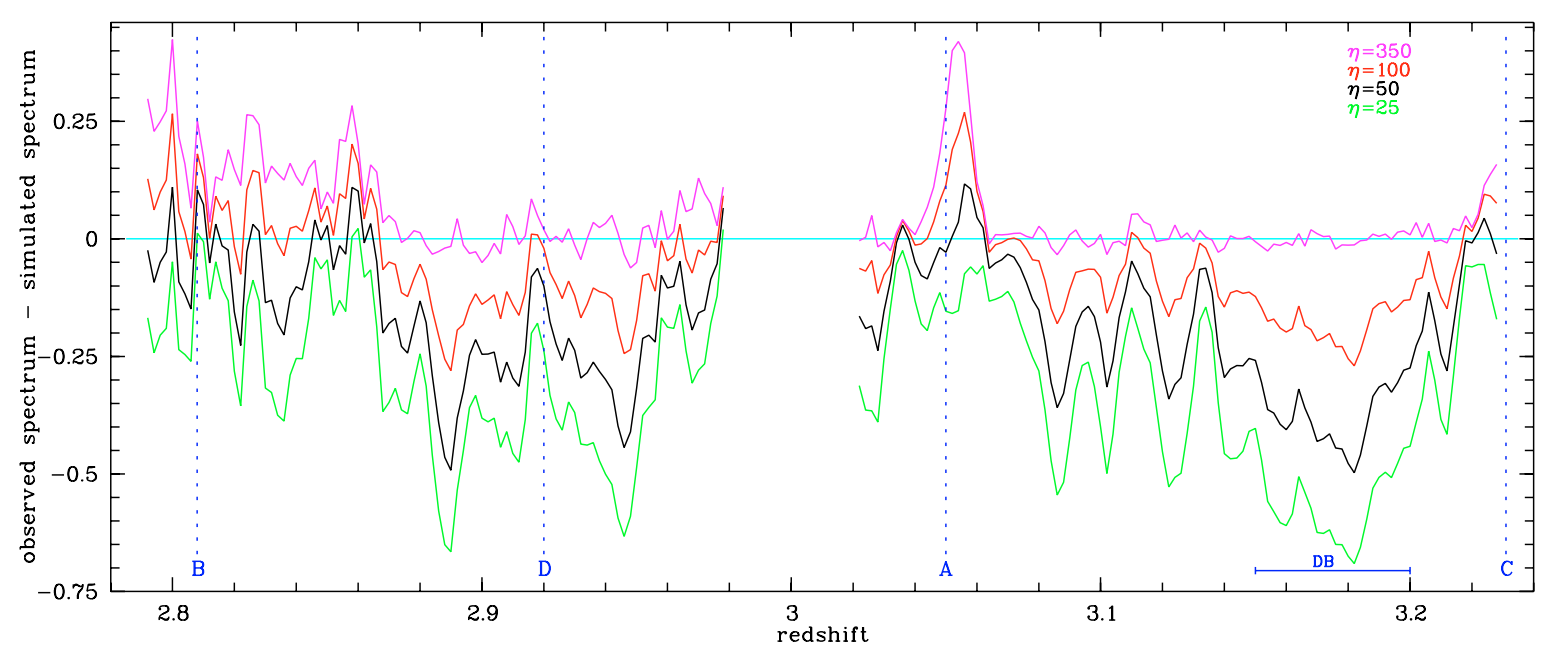

Fig. 8. Observed vs. predicted He II Ly $\alpha$ absorption, in differential representation (observed minus predicted). Different values for $\eta$ were used for the simulations (see text). The part of the spectrum with $z \leq 2.98$ was simulated using the H I line list by Hu et al. (1995), whereas the $\mathrm{H}$ I line list by Kim et al. (2002) was used at $z \geq 3.02$. The spectral region contaminated by geocoronal Ly $\alpha$ emission is not shown. Quasar and void locations are marked as in Fig. 4. Positive (negative) deviations from zero indicate that $\eta$ has to be smaller (higher) than the assumed $\eta$ of the curve. [See the online edition of the Journal for a colour version of this figure.]

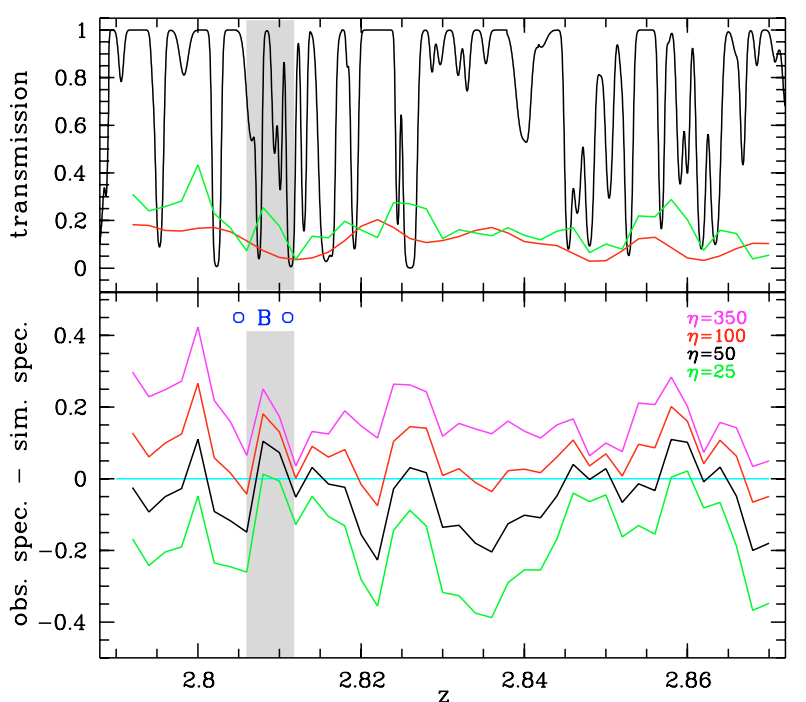

Fig. 9. Close-up of region B $(z<2.87)$. The upper panel shows the $\mathrm{H}$ I forest spectrum of Q 0302-003 reconstructed from the line list by $\mathrm{Hu}$ et al. (1995) (black), the He II transmission (green) and the simulated STIS spectrum for $\eta=100$ (red). The lower panel shows the fluctuating spectral hardness in this region (close-up of Fig. 8). The blue letter marks the foreground quasar QSO 03027-0010 (QSO B) at $z=2.808$. Blue circles indicate the two nearby LBGs found by Steidel et al. (2003). In the grey-shaded redshift range $2.806 \leq z \leq 2.812$, $\eta=25-50$ provides the best fit. [See the online edition of the Journal for a colour version of this figure.]

soft radiation field in the Gunn-Peterson trough can probably be attributed to the progressing He II reionization and the onset of a proper He II Lyman forest (Zheng et al. 2004b).

Notably, $\eta \simeq 25$ is reached only in two points, one of which precisely concides with the redshift where our newly discovered QSO B is located. This match is very unlikely to be a chance occurence; it is much more plausible to assume that hard radiation from QSO B intercepts the line of sight towards Q 0302-003 at this point.

Steidel et al. (2003) found two LBGs at angular distances of $\vartheta=3.04$ and $\vartheta=4.33$ from Q 0302-003 near the redshift of QSO B, at $z=2.805$ and $z=2.811$ (also indicated in Fig. 9). While the HI Lyman continuum escape fraction of LBGs is still debated (Steidel et al. 2001; Giallongo et al. 2002; Fernández-Soto et al. 2003; Shapley et al. 2003), the number of He II ionizing photons produced is at most a few per cent of the number of photons exceeding the H I Lyman edge according to current evolutionary synthesis models of starburst galaxies (Leitherer et al. 1999; Smith et al. 2002; Schaerer 2003). We therefore rule out the possibility that these two LBGs supply the hard photons required for the inferred $\eta$ minimum.

The situation is less clear for the weak QSO D also discovered by Steidel et al. (2003). At the redshift of this QSO, the overall appearance of the He II forest is that of a completely opaque Gunn-Peterson trough, probably reflecting a still partly neutral He II component of the IGM. This region is well described with $\eta \sim 350$ (Fig. 8). However, we see also that Fig. 8 suggests a drop down to $\eta \sim 100$ again at exactly the redshift of a QSO near the line of sight. This corresponds to a small spike of excess flux in the Gunn-Peterson trough (see Fig. 5) which is formally still consistent with the noise level. While we therefore cannot really claim to have detected another case of the transverse proximity effect, we cannot exclude it either, and the coincidence is certainly suggestive.

\section{Discussion}

Within the redshift range covered by the STIS data on the He II forest of Q 0302-003, five QSOs are known in or along the line of sight. Only one of these, the central quasar Q 0302-003 itself, shows unambiguous evidence for a proximity effect in HI. There is a possible small void near QSO A, 
but this could just as well be due to the overall distribution of $\mathrm{H}$ I material along the line of sight. At the redshifts of all three other QSOs, the transmission of the H I Lyman forest in the line of sight towards Q 0302-003 seems to be rather below average. Our estimated UV luminosities of these QSOs confirm that a classical transverse proximity effect is not really expected.

Considering the STIS UV spectra of the He II Lyman forest region instead, this picture does not change dramatically. Only in one case, at the redshift of QSO A, we see a clear "He II void", which in fact led to the discovery of the QSO (H00, J03). For QSO B and maybe also for QSO D we find nonzero He II transmissions, but these certainly do not qualify as voids in their own right. A similar case holds for QSO C (see Fig. 5), where however a confusion with the line of sight proximity effect zone of Q 0302-003 complicates the situation.

Only by combining H I and He II transmission properties, the radiative imprints of the QSOs along the line of sight become clearly separated from large scale fluctuations of the distribution of matter. This is particularly prominent for region $\mathrm{B}$ and QSO B, where the $\mathrm{H} I$ forest of Q 0302-003 shows in fact a complex of enhanced H I absorption, possibly even associated with the QSO in form of a large scale structure filament (Fig. 9). Eleven absorbers with $\log N_{\mathrm{H}_{\mathrm{I}}}>13$ reside in the redshift range $2.80<z<2.82$, four of them between the projected positions of the LBGs at $z=2.805$ and $z=2.811$. Assuming an average spectral hardness in this redshift regime $(\eta \sim 100)$, one expects strong corresponding He II absorption arising from these absorbers even at the low resolution of STIS (Fig. 9). Instead, we see that the He II forest remains transparent at this redshift, however with nothing that could be interpreted as a "void". Both spectral hardness estimators $R$ and the best-fit $\eta$, on the other hand, show a clearly pronounced extremum at precisely the correct redshift, in excellent agreement with the easier to interpret cases of QSO A and Q 0302-003. Thus, the most likely reasons for the different appearance of the He II transmission portions near QSO A (He II void) and QSO B (no void) in spite of a similar inferred hardness are different intrinsic densities of the overionized regions (small H I void vs. filament) combined with the progressing He II reionization with lower fluctuations of the He II transmission and the spectral hardness at lower redshift. The same effect, though much less significant, can be seen for QSO D.

Even for QSO C there might be a similar imprint onto the Q 0302-003 line of sight. Given the UV luminosity of Q 0302-003, the He III zone in the line of sight is surprisingly large, especially if the He II abundance in the surrounding IGM was still high. A possible additional contributor of hard ionizing photons could be the very luminous QSO C, intercepting the low- $z$ end of the proximity effect region of Q 0302-003. Figures 7 and 8 provide evidence for a very hard radiation field at just the redshift of QSO C which then, towards Q 0302-003, becomes somewhat softer again before finally hardening in the inner proximity effect zone. We suggest that the He II void at $z>3.22$ is really generated by the combined actions of a transverse proximity effect of QSO C and a line of sight effect of Q 0302-003.
We conclude that there is strong evidence for a transverse proximity effect in spectral hardness occuring in several incidences along the line of sight towards Q 0302-003. While individual $\eta$ fluctuations might still be regarded as coincidental, and possibly due to the crude diagnostics used, local minima of $R$ and $\eta$ are in fact found at exactly the redshifts of all five relevant QSOs, indicating a UV radiation field significantly enhanced due to a local hard source. Notice that because of the low overall UV background at 4 ryd, the proximity effect zone in spectral hardness is considerably larger than that of the conventional $\mathrm{H}$ I proximity effect. For QSO B, it reaches over $\sim 5 \mathrm{Mpc}$, and for the luminous QSO C it may even bridge more than $10 \mathrm{Mpc}$.

Besides the continuum placement and the background subtraction, the main source of uncertainty in our assessment of $R$ and $\eta$ is the correction for weak unresolved H I lines, the "shallow H I Gunn-Peterson trough" created by the quasi-diffuse component in the IGM. Small changes in the adopted correction lead to large changes in the best-fit $\eta$, as $\eta$ is very sensitive to small H I column densities. The same is true for the optical depth ratio $R$, which is very sensitive at high H I transmission values. This degeneracy affects the inferred $R$ and $\eta$ values as such, but the observed fluctuations and their correlation with the redshifts of known quasars are largely unaffected. Furthermore, the range of our inferred $\eta$ values $(25 \lessgtr \eta \lesssim 350$ ) is generally believed to be typical for the conditions in the IGM at $z \sim 3$.

A very interesting, though highly speculative observation concerns the relation between the strength of the "spectral hardness proximity effect" and the UV spectral slope of the QSO in question. QSO A is about 1 mag brighter in $V$ than QSO B, and it is also somewhat closer to the central line of sight. On the other hand, the inferred $\eta$ is even slightly lower at the redshift of QSO B than in void A. Adopting the continuum slopes given in Table 4 and boldly extrapolating towards 4 ryd, we predict values of the spectral softness of $S \simeq 29$ for QSO A and $S \simeq 10$ for QSO B; thus, the latter QSO is expected to be even brighter at the He II ionizing limit than the former. The higher $\eta$ values are therefore inferred near QSO A which has the softer spectrum, whereas lower $\eta$ values fit the data near QSO B which has the harder spectrum.

\section{A lower limit on the quasar lifetime}

Both incarnations of the proximity effect allow a lower limit on the quasar lifetime $t_{\mathrm{q}}$, since photons from a quasar need a certain time to propagate to the point of their absorption in the IGM. The observation of the line-of-sight proximity effect in the Ly $\alpha$ forest yields only lower limits based on the typical equilibrium timescale of $\operatorname{Ly} \alpha$ absorbers of $\simeq 10^{4}$ yr (e.g. Bajtlik et al. 1988), mainly because the line-of-sight proximity effect observed in a line forest is a statistical effect. However, in a mostly neutral medium, there exists the sharp observable boundary that defines the radius of the Strömgren sphere around the quasar. So far, this has been observed in H I Ly $\alpha$ towards SDSS J1030+0524 at $z=6.28$ (Pentericci et al. 2002) and in He II Ly $\alpha$ towards Q 0302-003 (Hogan et al. 1997) and PKS 1935-692 (Anderson et al. 1999). Simple modelling 
Table 5. Minimum lifetimes $\Delta t^{\prime}=d_{\perp} / c$ for all foreground quasars of Q 0302-003 with an indication of the transverse proximity effect.

\begin{tabular}{lllll}
\hline \hline QSO & Abbr. & $z$ & $d_{\perp}[\mathrm{Mpc}]$ & $\Delta t^{\prime}[\mathrm{Myr}]$ \\
\hline QSO 03027-0010 & B & 2.808 & 5.29 & 17 \\
QSO 0302-D113 & D & 2.920 & 2.26 & 7 \\
QSO 03020-0014 & A & 3.050 & 2.97 & 9 \\
Q 0301-005 & C & 3.231 & 10.34 & 34 \\
\hline
\end{tabular}

of the zone inside the Strömgren sphere yields $t_{\mathrm{q}} \gtrsim 13 \mathrm{Myr}$ (Pentericci et al. 2002) and $t_{\mathrm{q}} \gtrsim 40 \mathrm{Myr}$ (Hogan et al. 1997).

In the case of the transverse proximity effect one cannot measure the extent of the proximity effect zone, neither in a line forest nor in a Gunn-Peterson trough, since the proximity effect zone is intersected by another line of sight by chance. The emission epoch of the ionizing photons of the source in the direction of the background line of sight cannot be observed, since these photons have to be emitted prior to our observations. So the transverse proper distance $d_{\perp}$ between the two lines of sight at the emission redshift of the foreground quasar has to serve as an approximation for the expanding distance between the source and the emitted photons. Due to the expansion of the universe between the emission and the absorption of the photons, the inferred light travel time $\Delta t^{\prime}=d_{\perp} / c$ is larger than the actual light travel time $\Delta t$, but usually the correction for expansion is very small.

We have argued in the previous section that the QSOs A-D show at least an indication for the transverse proximity effect in the line of sight towards Q 0302-003. Assuming a correspondence between the known foreground quasars and He III zones around them having low $\eta$, we can infer their minimum total quasar lifetimes listed in Table 5. These are all consistent with the estimates based on the proximity effect (Srianand 1997; Hogan et al. 1997; Anderson et al. 1999; Pentericci et al. 2002; J03) and theoretical arguments (Martini 2004, and references therein). Due to the high sensitivity of the spectral hardness we are able to constrain the quasar lifetime for a sample of quasars forming a close group on the sky, yielding minimum quasar lifetimes in the range $\Delta t^{\prime} \gtrsim 10-30 \mathrm{Myr}$.

\section{Conclusions}

The transverse proximity effect in quasar spectra has so far mostly been associated with the notion of voids in the H I Lyman forest, created by the overionized zones around quasars near the line of sight. It has always been clear, however, that true voids due to large scale structure and apparent voids due to the proximity effect would be essentially indistinguishable on an individual basis. This problem holds also for the He II Lyman forest that has now become accessible for a small number of high-redshift QSOs.

We argue in this study that spectral hardness indicators provide an efficient way to discriminate between large scale structure and proximity effect. The line of sight proximity effect towards Q 0302-003 and the transverse proximity effect due to QSO 03020-0014 (dubbed "QSO A" in this paper) were already discussed by Heap et al. (2000) and Jakobsen et al. (2003) and are, since the discovery of QSO A, clear-cut cases where both the optical depth ratio and the hardness parameter $\eta$ show pronounced minima close to the quasars. We investigated the $\mathrm{H}$ I and $\mathrm{He}$ II absorption properties at redshifts near our newly discovered QSO 03027-0010 (“QSO B”), and also near the other known QSOs close to the line of sight. In each single case we find evidence for a link between spectral hardness and the presence of a nearby quasar.

Thus, the relative UV hardness is a sensitive physical quantity to search for individual sources of the metagalactic UV radiation field beyond the simple detection of associations between quasars and voids. Quasars and voids may be unrelated, whereas the spectral hardness is related to the spectral energy distributions of the ionizing sources. In particular, a void will not occur if the quasar overionizes an intrinsically overdense region. The spectral hardness may break this density degeneracy that affects studies of the proximity effect (Loeb \& Eisenstein 1995; Schirber et al. 2004; Rollinde et al. 2005). At least for QSO B, this case of an overdense region is apparently applicable.

The excellent match between the measured QSO redshifts and the inferred minima of $\eta(z)$ imply that there is essentially no need to invoke strongly anisotropic radiation for any of the quasars in question. This is in stark contrast to the situation for the H I proximity effect where anisotropy or even beaming has repeatedly been claimed (Dobrzycki \& Bechtold 1991a; Crotts 1989; Møller \& Kjærgaard 1992; Schirber et al. 2004). It is likely that true large scale structure in the Lyman forest has contributed to mask the intrinsic transverse proximity effect in several of these cases.

The redshift range of $2.7 \lesssim z<3.2$ studied in the line of sight towards Q 0302-003 is of very high interest in the context of studying the putative reionization epoch of He II. We have shown that one can determine the sources that ionize this line of sight, even at low resolution. Near the low-redshift end of the covered spectral range, there are clear indications for a transition from He III bubbles in the IGM towards a more or less fully ionized He III IGM where also the overall UV background is dominated by QSO radiation. Our estimates of $\eta$ in this redshift range are fully compatible with this concept. Even then, local sources of very hard radiation such as QSO B can significantly modify the UV background over distances of several Mpc. It would be highly desirable to be able to study these processes at higher spectral resolution. Unfortunately, Q 0302-003 is too faint for observations with the Far Ultraviolet Spectroscopic Explorer, and the only perspective for significantly improved data lies with the Cosmic Origins Spectrograph to be installed on HST.

Acknowledgements. We thank the staff of the ESO observatories La Silla and Paranal for their professional assistance in obtaining the optical data discussed in this paper. We are grateful to Aldo Dall' Aglio for providing his code to simulate absorption line spectra from a given line list. G.W. acknowledges support by a HWP grant from the state of Brandenburg, Germany.

Funding for the creation and distribution of the SDSS Archive has been provided by the Alfred P. Sloan Foundation, the Participating Institutions, the National Aeronautics and Space Administration, 
the National Science Foundation, the US Department of Energy, the Japanese Monbukagakusho, and the Max Planck Society. The SDSS Web site is http://www.sdss.org/. The SDSS is managed by the Astrophysical Research Consortium (ARC) for the Participating Institutions. The Participating Institutions are The University of Chicago, Fermilab, the Institute for Advanced Study, the Japan Participation Group, The Johns Hopkins University, the Korean Scientist Group, Los Alamos National Laboratory, the MaxPlanck-Institute for Astronomy (MPIA), the Max-Planck-Institute for Astrophysics (MPA), New Mexico State University, University of Pittsburgh, University of Portsmouth, Princeton University, the United States Naval Observatory, and the University of Washington.

\section{References}

Adelberger, K. L., Steidel, C. C., Shapley, A. E., \& Pettini, M. 2003, ApJ, 584, 45

Anderson, S. F., Hogan, C. J., Williams, B. F., \& Carswell, R. F. 1999, AJ, 117, 56

Bajtlik, S., Duncan, R. C., \& Ostriker, J. P. 1988, ApJ, 327, 570

Barbieri, C., \& Cristiani, S. 1986, A\&AS, 63, 1

Bryan, G. L., Machacek, M., Anninos, P., \& Norman, M. L. 1999, ApJ, 517, 13

Croft, R. A. C. 2004, ApJ, 610, 642

Croft, R. A. C., Weinberg, D. H., Pettini, M., Hernquist, L., \& Katz, N. 1999, ApJ, 520, 1

Crotts, A. P. S. 1989, ApJ, 336, 550

Crotts, A. P. S., \& Fang, Y. 1998, ApJ, 502, 16

Davidsen, A. F., Kriss, G. A., \& Zheng, W. 1996, Nature, 380, 47

Dobrzycki, A., \& Bechtold, J. 1991a, ApJ, 377, L69

Dobrzycki, A., \& Bechtold, J. 1991b, in The Space Distribution of Quasars, ASP Conf. Ser., 21, 272

Fardal, M. A., \& Shull, J. M. 1993, ApJ, 415, 524

Fardal, M. A., Giroux, M. L., \& Shull, J. M. 1998, AJ, 115, 2206

Fernández-Soto, A., Barcons, X., Carballo, R., \& Webb, J. K. 1995 , MNRAS, 277, 235

Fernández-Soto, A., Lanzetta, K. M., \& Chen, H.-W. 2003, MNRAS, 342,1215

Gaskell, C. M. 1982, ApJ, 263, 79

Giallongo, E., Cristiani, S., D’Odorico, S., \& Fontana, A. 2002, ApJ, 568, L9

Gunn, J. E., \& Peterson, B. A. 1965, ApJ, 142, 1633

Haardt, F., \& Madau, P. 1996, ApJ, 461, 20

Heap, S. R., Williger, G. M., Smette, A., et al. 2000, ApJ, 534, 69

Hernquist, L., Katz, N., Weinberg, D. H., \& Miralda-Escudé, J. 1996, ApJ, 457, L51

Hogan, C. J., Anderson, S. F., \& Rugers, M. H. 1997, AJ, 113, 1495

Horne, K. 1986, PASP, 98, 609

Hu, E. M., Kim, T.-S., Cowie, L. L., \& Songaila, A. 1995, AJ, 110, 1526

Jakobsen, P., Boksenberg, A., Deharveng, J. M., et al. 1994, Nature, 370,35

Jakobsen, P., Jansen, R. A., Wagner, S., \& Reimers, D. 2003, A\&A, 397, 891

Kim, T.-S., Carswell, R. F., Cristiani, S., D’Odorico, S., \& Giallongo, E. 2002, MNRAS, 335, 555

Kim, T.-S., Hu, E. M., Cowie, L. L., \& Songaila, A. 1997, AJ, 114, 1

Kriss, G. A., Shull, J. M., Oegerle, W., et al. 2001, Science, 293, 1112

Leitherer, C., Schaerer, D., Goldader, J. D., et al. 1999, ApJS, 123, 3

Liske, J. 2000, MNRAS, 319, 557

Liske, J., \& Williger, G. M. 2001, MNRAS, 328, 653
Loeb, A., \& Eisenstein, D. J. 1995, ApJ, 448, 17

Madau, P. 1995, ApJ, 441, 18

Martini, P. 2004, in Carnegie Observatories Astrophysics Series, Vol. 1: Coevolution of Black Holes and Galaxies, ed. L. C. Ho (Cambridge University Press), 170

McDonald, P., Seljak, U., Cen, R., Bode, P., \& Ostriker, J. P. 2005, MNRAS, 360, 1471

McIntosh, D. H., Rix, H.-W., Rieke, M. J., \& Foltz, C. B. 1999, ApJ, 517, L73

Meiksin, A., \& White, M. 2004, MNRAS, 350, 1107

Møller, P., \& Jakobsen, P. 1990, A\&A, 228, 299

Møller, P., \& Kjærgaard, P. 1992, A\&A, 258, 234

Pentericci, L., Fan, X., Rix, H.-W., et al. 2002, AJ, 123, 2151

Reimers, D., Köhler, S., Wisotzki, L., et al. 1997, A\&A, 327, 890

Reimers, D., Fechner, C., Kriss, G., et al. 2004 [arXiv: astro-ph/0410588]

Reimers, D., Fechner, C., Hagen, H.-J., et al. 2005, A\&A, 442, 63

Ricotti, M., Gnedin, N. Y., \& Shull, J. M. 2000, ApJ, 534, 41

Rollinde, E., Srianand, R., Theuns, T., Petitjean, P., \& Chand, H. 2005, MNRAS, 361, 1015

Sargent, W. L. W., Steidel, C. C., \& Boksenberg, A. 1989, ApJS, 69, 703

Schaerer, D. 2003, A\&A, 397, 527

Schaye, J., Theuns, T., Rauch, M., Efstathiou, G., \& Sargent, W. L. W. 2000, MNRAS, 318, 817

Schirber, M., Miralda-Escudé, J., \& McDonald, P. 2004, ApJ, 610, 105

Scott, J., Bechtold, J., Dobrzycki, A., \& Kulkarni, V. P. 2000, ApJS, 130,67

Shapley, A. E., Steidel, C. C., Pettini, M., \& Adelberger, K. L. 2003, ApJ, 588, 65

Shull, J. M., Tumlinson, J., Giroux, M. L., Kriss, G. A., \& Reimers, D. 2004, ApJ, 600, 570

Smette, A., Heap, S. R., Williger, G. M., et al. 2002, ApJ, 564, 542

Smith, L. J., Norris, R. P. F., \& Crowther, P. A. 2002, MNRAS, 337, 1309

Songaila, A., Hu, E. M., \& Cowie, L. L. 1995, Nature, 375, 124

Srianand, R. 1997, ApJ, 478, 511

Steidel, C. C., Pettini, M., \& Adelberger, K. L. 2001, ApJ, 546, 665

Steidel, C. C., Adelberger, K. L., Shapley, A. E., et al. 2003, ApJ, 592, 728

Telfer, R. C., Zheng, W., Kriss, G. A., \& Davidsen, A. F. 2002, ApJ, 565,773

Theuns, T., Zharoubi, S., Kim, T.-S., Tzanavaris, P., \& Carswell, R. F. 2002, MNRAS, 332, 367

Tytler, D., \& Fan, X. 1992, ApJS, 79, 1

Véron-Cetty, M.-P., \& Véron, P. 2003, A\&A, 412, 399

Weinberg, D. H., Hernquist, L., Katz, N., Croft, R., \& Miralda-Escudé, J. 1997, in Proc. of the 13th IAP Astrophysics Colloquium: Structure and Evolution of the Intergalactic Medium from QSO Absorption Line System, ed. P. Petitjean, \& S. Charlot (Paris: Editions Frontières), 133

Wisotzki, L., Selman, F., \& Gilliotte, A. 2001, The Messenger, 104, 8 Zhang, Y., Anninos, P., \& Norman, M. L. 1995, ApJ, 453, L57

Zhang, Y., Meiksin, A., Anninos, P., \& Norman, M. L. 1998, ApJ, 495, 63

Zheng, W., Chiu, K., Anderson, S. F., et al. 2004a, AJ, 127, 656

Zheng, W., Kriss, G. A., Deharveng, J.-M., et al. 2004b, ApJ, 605, 631

Zuo, L. 1992, MNRAS, 258, 36

Zuo, L. 1993, A\&A, 278, 343 